\title{
Lidil
}

Revue de linguistique et de didactique des langues

$52 \mid 2015$

Les pratiques artistiques dans l'apprentissage des langues

\section{Vers une articulation entre didactique de la littérature, pratique théâtrale et approche interactionnelle}

Towards an Articulation between Teaching of Literature, Drama and

Interaction-Based Approach

\section{Christian Ollivier}

\section{OpenEdition}

\section{Journals}

Édition électronique

URL : http://journals.openedition.org/lidil/3802

DOI : $10.4000 /$ lidil.3802

ISSN : 1960-6052

\section{Éditeur}

UGA Éditions/Université Grenoble Alpes

\section{Édition imprimée}

Date de publication : 20 novembre 2015

Pagination : 15-37

ISBN : $978-2-84310-312-4$

ISSN : $1146-6480$

\section{Référence électronique}

Christian Ollivier, « Vers une articulation entre didactique de la littérature, pratique théâtrale et approche interactionnelle », Lidil [En ligne], 52 | 2015, mis en ligne le 01 janvier 2017, consulté le 30 octobre 2020. URL : http://journals.openedition.org/lidil/3802 ; DOI : https://doi.org/10.4000/lidil.3802 


\title{
Vers une articulation entre didactique de la littérature, pratique théâtrale et approche interactionnelle
}

\author{
Christian Ollivier*
}

\begin{abstract}
RÉsumÉ
Après avoir exposé un positionnement théorique posant toute communication comme processus dialogique de co-énonciation et faisant le lien avec des théories actuelles de la littérature, nous proposons un modèle didactique centré sur des tâches issues du monde du théâtre. Nous montrons qu'elles permettent d'allier de façon intégrée une approche socio-interactionnelle du texte de théâtre avec des objectifs d'ordre linguistique, langagiers et (inter)culturels.
\end{abstract}

\begin{abstract}
In this paper, we first present our theoretical position from a linguistic and literary point of view; assuming that communication as well as literary reading are dialogical processes of co-construction of meaning. On this basis we propose a didactical model centred on the use of theatre-tasks. We show that this kind of tasks allows building learning units that combine, in an integrative way, a socio-interactional approach of theatre texts and linguistic, communicative and (inter)cultural objectives.
\end{abstract}

Ce texte entend proposer une réflexion argumentée autour de la possible articulation entre didactique de la littérature, pratique théâtrale et approche interactionnelle.

\section{CECRL et littérature}

À la lecture du Cadre européen commun de référence pour les langues (Conseil de l'Europe, 2001; désormais CECRL), cette articulation peut

* Professeur des universités en didactique des langues/sciences du langage, Université de La Réunion, laboratoire LCF-Icare, département de FLE. 
ne pas paraitre évidente. La littérature n'y trouve en effet qu'une place limitée. Dans les descriptions des niveaux communs de référence, la lecture de textes littéraires n'apparait qu'au niveau B2, avec une restriction aux œuvres contemporaines et écrites en prose : «je peux comprendre un texte littéraire contemporain en prose» (ibid., p. 27). La littérature se retrouve également dans la partie consacrée à l'«utilisation esthétique ou poétique de la langue» qui - soit dit en passant recense deux fois le théâtre en quelques lignes évoquant d'une part parmi les «activités», «le théâtre (écrit ou improvisé)» et, d'autre part, «la production, la réception et la représentation de textes littéraires comme - lire et écrire des textes (nouvelles, romans, poèmes, etc.) représenter et regarder ou écouter un récital, un opéra, une pièce de théâtre, etc.». Les auteurs du CECRL sont conscients de n'accorder que peu d'espace à la littérature et se défendent d'y voir un traitement «un peu cavalier» de ce qui «a traditionnellement été un aspect important, souvent essentiel, des études de langue vivante au secondaire et dans le supérieur». Ils soulignent la diversité des finalités des études littéraires qui dépassent la seule dimension esthétique et expriment l'espoir que «les professeurs de littérature à tous les niveaux trouvent que des sections du Cadre de référence sont pertinentes pour eux et utiles en ce qu'elles rendent leurs buts et leurs démarches plus transparents» (ibid., p. 47). Finalement, la capacité à enseigner la littérature se retrouve également classée dans les «approches particulières» (ibid., p. 111) au même titre que la «capacité à individualiser l'enseignement», «l'aptitude à tenir la classe», «la capacité à faire de la recherche et à prendre des distances par rapport à son expérience», par exemple. De cette lecture du CECRL, il ressort que le travail sur les textes littéraires est réservé aux niveaux supérieurs et que la littérature, en général, et le texte littéraire, en particulier, semblent être conçus comme pouvant (devant?) faire l'objet d'une approche spécifique, impression que renforce la distinction effectuée (cf. supra) entre le professeur de langues et le professeur de littérature.

Dans le monde de la didactique des langues, l'intérêt pour les pratiques artistiques trouve son écho dans plusieurs colloques et ouvrages ou numéros de revue récents - notamment Alix, Lagorgette \& RollinatLevasseur, 2013; Cornaz \& Fonio, 2014; Payet, 2010 ; SpangheroGaillard \& Garnier, 2014. Quelques articles se sont ainsi penchés sur une approche du texte littéraire dans une perspective actionnelle (par exemple Bemporad, 2010; Luscher, 2009; Puren, 2006 et 2012), d'autres, plus rares, sur les pratiques théâtrales et l'actionnel (par exemple Cornaz 
\& Fonio, 2014; Fonio \& Genicot, 2011; Morel, 2014). Mais il existe, au final, encore peu de publications traitant spécifiquement de l'articulation entre les trois pôles que constituent la didactique de la littérature, les pratiques théâtrales et la perspective actionnelle dans un enseignement-apprentissage des langues faisant appel à des tâches issues du monde du théâtre - nous entendons par là des tâches que réalisent les professionnels du théâtre dans leur formation ou l'exercice de leur métier.

\section{Positionnement théorique}

\subsection{Dimension linguistique}

Nous situant dans une approche socio-interactionnelle, nous considérons que toute réception - qu'il s'agisse ou non de textes littéraires est un acte dialogique impliquant, au minimum, le scripteur et le lecteur. Nous partons du principe que toute communication et donc toute lecture est une co-construction de sens sous contrainte relationnelle et reprenons ainsi la notion de primum relationis largement développée par Jacques (1979, 1982, 1985, 2000 notamment) et Grillo (2000). Plus encore que le dialogisme bakhtinien (Bakhtine, 1978 et 1984), cette pensée revient à cesser de considérer d'un côté un émetteur (ou locuteur) et de l'autre côté un récepteur (ou allocutaire) «alternativement protagonistes», comme les concevait Benveniste (1974, p. 85) pour voir dans les partenaires de la communication des co-énonciateurs partageant l'initiative sémantique. En cela, tout énoncé est plurivocal - pas seulement au sens intertextuel de Bakhtine (1984) ou d'autres spécialistes de l'intertextualité tels que Kristeva ou Genette - mais parce que «tout se passe comme si en chaque énonciation on entendait deux voix ${ }^{1}$ » (Jacques, 2000, p. 69), voire plus. Dans un tel modèle, «le moi et le toi sont [...] mutuellement associés à l'intérieur d'une communauté de communication. Aucune réciprocité véritable du Je au Tu, aucune complémentarité entre leurs rôles ne se maintient durablement sans la médiation qui les unit en "nous" (Jacques, 1979, p. 386). Il en découle que signifier et comprendre sont des activités conjointes et que le sémantisme des énoncés est déterminé par l'interaction sociale au sein de laquelle ils prennent place. Il n'y a plus, d'un côté, un émetteur qui coderait un à-dire préexistant et, d'un autre côté, un récepteur

1. Italiques dans le texte original. 
qui décoderait - «passivement», dirait Bakhtine (1984) - le message pour reconstituer cet à-dire originel. Dans une pensée socio-interactionnelle, «S1 et S2 [= les sujets énonciateurs] collaborent par actes de langage et confrontation épistémique, pour produire $x$ comme signe de $y$ » (Jacques, 1985, p. 209). Ou, dans les termes de Grillo (1997) : «Dès lors, parler n'est plus seulement dire quelque chose à quelqu'un : c'est bien plus fondamentalement, dire, à propos de quelque chose, quelque chose avec quelqu'un ${ }^{2} . »($ p. 63)

La conséquence est que le sens n'existe pas en soi et antérieurement à tout acte de langage, il est le fruit d'une co-construction et n'apparait que dans l'interaction. C'est «le "dire avec autrui" qui précède tout sens» (Jacques, 2000, p. 49). Ou plutôt, en accord avec Derrida (1967) qui, se fondant sur l'«irréférence au centre» du signe, pense que «le sens n'est ni avant ni après l'acte» (p. 22), c'est dans le dire avec autrui qu'émerge le sens. Car, comme le formule Culioli (1999), «l'énoncé n'a pas de sens sans une double intention de signification chez les énonciateurs respectifs» (p. 47), ou encore : «le texte n'a de sens en dehors de l'activité signifiante des énonciateurs» (p. 48). L'initiative sémantique n'est donc plus de la seule responsabilité du locuteur ni de celle du destinataire, elle est partagée et le fruit d'une interaction entre deux instances énonciatives. Penser cela, c'est remettre en cause l'idée d'un sens prédéterminé accessible aux interlocuteurs. C'est donc, en didactique de la littérature, écarter les approches qui visent à rechercher le sens des textes.

\subsection{Dimension littéraire}

Cette façon d'appréhender la communication et la construction du sens est proche des théories littéraires qui ont mis à mal l'idée d'un sens préalable à l'écriture. Mallarmé (1897, p. 246) annonçait ainsi la «disparition élocutoire du poëte». Valéry, s'opposant directement à l'idéologie romantique du génie inspiré, voyait, en 1941 dans Rhumbs, le texte littéraire comme «l'œuvre de bien d'autres choses qu'un "auteur" » (Valéry, 1960, p. 629) et décrivait un écrivain travaillant (un "constructeur») dont l'œuvre est faite de tâtonnements, d'hésitations, de corrections incessantes, ce qui est écrit, ce qui est «fait» étant plus important que ce que l'auteur voulait dire. Dans «Au sujet du Cimetière marin», Valéry remettait ainsi en cause l'existence d'un «vrai sens» du texte,

2. Les surlignements sont d'Éric Grillo. 
le sens n'étant plus à chercher du côté de l'auteur, mais survenant lors de la lecture, celle de l'auteur n'étant pas supérieure à celle d'un autre lecteur :

Il n'y a pas de vrai sens d'un texte. Pas d'autorité de l'auteur. Quoi qu'il ait voulu dire, il a écrit ce qu'il a écrit. Une fois publié, un texte est comme un appareil dont chacun peut se servir à sa guise et selon ses moyens : il n'est pas sûr que le constructeur en use mieux qu'un autre. Du reste, s'il sait bien ce qu'il voulût faire, cette connaissance trouble toujours en lui la perception de ce qu'il a fait. (Valéry, 1933, p. 35)

Barthes défend des prises de position semblables. En 1968, dans un texte éponyme, il déclare «la mort de l'auteur» et remet en cause le principe selon lequel «l'explication de l'œuvre est toujours cherchée du côté de celui qui l'a produite, comme si [...] c'était toujours finalement la voix d'une seule et même personne, l'auteur, qui livrait sa "confidence"» (Barthes, 1994, p. 92 et suiv.). Or, poursuit le philosophe : «le texte n'est pas fait d'une ligne de mots, dégageant un sens unique, en quelque sorte théologique (qui serait le "message" de l'Auteur-Dieu)» (ibid., p. 94 et suiv.). Abandonnant une approche centrée sur l'auteur et la reconstitution d'un sens auctorial, Barthes se tourne alors vers le lecteur, car «l'unité d'un texte n'est pas dans son origine, mais dans sa destination» (ibid., p. 495).

En résumé, l'écriture (littéraire) n'est pas la transcription d'un à-dire antérieur, elle est un faire, le sens n'étant plus préexistant au texte. Si le sens n'est pas à chercher du côté de l'auteur, on pourrait être tenté de le chercher du côté du lecteur, comme semble le dire Barthes dans le texte que nous venons de citer, mais les théories littéraires des années 1980 le situent dans l'interaction entre auteur, texte et lecteur.

Eco (1985) conçoit la lecture littéraire comme une «coopération interprétative» - entre lecteur et auteur idéal essentiellement, mais aussi entre lecteur et auteur empiriques (Eco, 2013) - dans laquelle le lecteur remplit les espaces vides du texte, ce en quoi il rejoint Iser (1976), un des pères de l'École de Constance, qui pose que le lecteur est d'emblée inclus dans le processus de production de l'œuvre littéraire, l'auteur laissant des «Leerstellen» (places vides) que le lecteur est appelé à compléter. Dans ce genre de modèle, l'auteur place dans son texte des indices censés aider le lecteur à co-construire du sens.

Eco pose la multiplicité des sens potentiels qui peuvent surgir de la lecture littéraire. Dans les Postille a "Il nome della rosa" (Eco, 1983), il considère qu' «un narratore non deve fornire interpretazioni della 
propria opera» puisqu'un roman est «una macchina per generare interpretazioni $\gg$ et précise que «nulla consola maggiormente un autore di un romanzo che lo scoprire letture a cui egli non pensava, e che i lettori gli suggeriscono $»^{3}$.

Sartre avait déjà largement théorisé cette vision quand, dans Qu'estce que la littérature? (1948), il soulignait l'aspect «incomplet et abstrait» de l'œuvre littéraire et évoquait le rôle éminent du lecteur. D'après lui, en effet, l'œuvre littéraire ne voit le jour comme objet que dans l'acte dialogique que constitue la lecture :

L'opération d'écrire implique celle de lire comme son corrélatif dialectique et ces deux actes connexes nécessitent deux agents distincts. C'est l'effort conjugué de l'auteur et du lecteur qui fera surgir cet objet concret et imaginaire qu'est l'ouvrage de l'esprit. Il n'y a d'art que pour et par autrui. (Sartre, 1948, p. 93)

Le rôle essentiel du lecteur est défini comme :

[...] requis non seulement pour dévoiler l'objet (c'est-à-dire faire qu'il y ait objet) mais encore pour que cet objet soit absolument (c'est-à-dire pour le produire). En un mot, le lecteur a conscience de dévoiler et de créer à la fois, de dévoiler en créant, de créer par dévoilement. (Ibid., p. 93-94)

La pensée de Gadamer, fondée sur celle de Schleiermacher, allait dans une direction semblable. Ce dernier reconnaissait en effet au lecteur une compréhension du texte qui n'est pas forcément moindre que celle de son auteur. Il voyait dans l'interprétation et la compréhension d'un texte un «art» (technè), autrement dit un acte de création, l'œuvre d'art étant conçue comme une abstraction qui n'existe vraiment qu'à travers le regard de l'autre. Elle est «etwas abstractes, und insofern ist sie nichts an und für sich, sondern wird erst durch die Beziehungen, die der Beschauer hineinlegt ${ }^{4} \gg$ (Schleiermacher, 1843, p. 682). Sur cette base, pour Gadamer (1975), la compréhension et l'interprétation d'un

3. Eco considère qu' «un narrateur n'a pas à fournir d'interprétations à son œuvre» puisque les romans sont «des machines à générer des interprétations» et précise que «rien ne console plus l'auteur d'un roman que de découvrir les lectures auxquelles il n'avait pas pensé et que les lecteurs lui suggèrent» (notre traduction).

4. «Quelque chose d'abstrait qui n'est rien en soi ni pour soi et ne se réalise vraiment complètement que dans les relations qu'y met celui qui la regarde.» (Notre traduction) 
texte littéraire n'excluent ni neutralité objective ni effacement du moi puisque toute compréhension est, au final, compréhension de soi.

Dans une approche similaire, Bayard (1998, p. 128) pose que le texte littéraire n'existe pas indépendamment de la subjectivité du lecteur car, en fonction de celui-ci, «ce ne sont pas les mêmes signes qui seront mobilisés selon les lectures, ou les mêmes significations des mêmes signes». Dès lors, il n'existe pas de «texte objectivable», mais «[c]'est le lecteur qui vient achever l'œuvre et refermer le monde qu'elle ouvre, et il le fait à chaque fois d'une manière différente», posant sur le texte une signification individuelle et idiosyncratique. Le lecteur crée ainsi son propre texte à travers ce que Langlade (2008, p. 46) nomme «activité fictionnalisante», considérant qu'il n'y a lecture littéraire que «lorsque l'activité créatrice - imageante et imaginante - du lecteur permet d'ancrer les propositions de l'œuvre dans la personnalité profonde, la culture intime, l'imaginaire de celui-ci». Cet investissement subjectif du lecteur dépasse de loin la complétion de ce qu'Iser nomme les «Leerstellen» et le travail de remplissage des espaces de «non-dit ou de déjà-dit restés en blanc» évoqué par Eco (1985, p. 27). La subjectivité, qualifiée d' «accidentelle» par Jouve, peut en effet survenir à tout moment en dehors des moments prévus pour son apparition; ainsi «aux configurations nécessairement subjectives viennent s'ajouter des configurations accidentellement subjectives» (Jouve, 2004, p. 108).

\section{Positionnement didactique}

Nous nous inscrivons donc dans une didactique de la littérature qui accorde toute sa place au lecteur prenant son plaisir, à ce «contrehéros» que Barthes (1973) décrit comme «l'abjection de notre société» dont «les tribunaux, l'école, l'asile, la conversation [...] feraient un étranger», cet individu «qui mélangerait tous les langages, fussent-ils réputés incompatibles; qui supporterait, muet, toutes les accusations d'illogisme, d'infidélité; qui resterait impassible devant l'ironie socratique (amener l'autre au suprême opprobre : se contredire) et la terreur légale». Nous nous inscrivons dans une didactique de la littérature qui conçoit la lecture littéraire comme une construction de sens socio-interactionnelle unissant auteur et lecteur, mais également les lecteurs. Il s'agit de sortir de la lecture analytique et d'un enseignement de la littérature formaliste et techniciste (Langlade, 2004; Rouxel, 2010) qui traite le texte comme un «objet d'explication» (Barthes, 1994, p. 1245) et place le lecteur dans une position d'observateur et d'analyste, qui ne 
lui laisse d'autre rôle et d'autre «droit règlementaire» (Foucault, 1969, p. 68) que celui d'être le récepteur d'une littérature transmise. Avec pour conséquence que, le sujet-lecteur étant largement exclu (Rouxel, 2007 et 2010; Todorov, 2007), l'apprenant, fréquemment, «se désengage et ne s'affirme pas face au texte» et montre des réactions de «[r]ésignation désabusée, dérision, indifférence, refus» (Rouxel, 2007, p. 87).

L'approche que nous retenons ouvre «un espace de liberté pour le sujet lecteur» (Rouxel, 2007, p. 67). Sans laisser de côté le dialogue entre le lecteur et l'auteur autour du texte (voir par exemple Bredella, 1980; Tauveron, 2004), elle entend «réhabiliter la subjectivité» (Rouxel, 2007, p. 89) en accordant une place importante au lecteur co-énonciateur et aux échanges entre apprenants pour favoriser la rencontre des subjectivités. Dans l'optique d'une «articulation entre réception personnelle et réception collective», telle qu'évoquée par Bernanoce (2013), les échanges entre lecteurs nous semblent en effet également devoir être encouragés car, d'une part, ils replacent l'activité littéraire dans un cadre social immédiat et d'autre part, ils peuvent, comme nous l'avons montré (Ollivier, 2009 et 2010), largement participer à la construction socio-interactionnelle du sens.

Nous nous situons également dans une approche actionnelle de la littérature, proche du sens allemand de «handlungsorientierter Literaturunterricht» (Eichmann, 1990 ; Gudjons, 1986; Haas, 2005 ; Menzel, 2000), et encore plus de celui de la perspective actionnelle. La didactique actionnelle de la littérature - généralement liée à une approche orientée vers la production esthético-artistique - met en avant l'activité esthétique de l'apprenant, qu'il s'agisse d'écriture ou d'autres formes d'expression artistique : peinture, photographie, mise en scène théâtrale, etc. En proposant des voies d'accès au texte se distinguant des approches analytiques, ces orientations tiennent compte des individualités en présence et de leur sensibilité, notamment artistique, et entendent développer l'imagination des apprenants conçue comme fondement de la lecture littéraire.

Le texte théâtral occupe une position particulière dans le spectre des textes littéraires car, autant les autres genres font essentiellement l'objet de lectures individuelles et potentiellement silencieuses (cf. «le droit de ne rien dire» de Pennac, 1992), autant le texte de théâtre donne généralement lieu à un travail conduisant à une représentation publique. Il s'agit d'un faire impliquant plusieurs personnes et mettant donc en jeu de nombreuses interactions sociales complexes. Ce travail en amont des représentations engage différents professionnels du théâtre. La re- 
présentation, quant à elle, peut être considérée comme le partage avec un public d'une co-interprétation du texte issue du travail effectué en amont.

Le lien entre l'approche actionnelle du CECRL et le théâtre peut sembler plutôt évident lorsqu'il s'agit justement de monter un projet théâtral débouchant sur une représentation, la pédagogie de projet et la perspective actionnelle présentant de nombreux points communs. Nous ne nous intéresserons cependant pas à ce genre de projets ${ }^{5}$, qui, comme le rappelle Bernanoce (2013), dépasse le cadre d'un cours normal, mais aux pratiques que l'on regroupe en pays germanophones sous le terme générique de Dramapädagogik par opposition à Theaterpädagogik, distinction reprise en partie du monde anglophone. Nous en retenons l'un des éléments distinctifs essentiels : la seconde est orientée-produit (même si le processus y joue un rôle important); elle vise la mise en scène d'un spectacle qui sera présenté à un public tandis que la Dramapädagogik - en anglais drama in education ou drama as education (voir Bolton, 1979 et 1984) et que l'on pourrait traduire en français par «pédagogie fondée sur le jeu dramatique» - est orientée-processus; elle vise des objectifs liés à la discipline enseignée et se sert d'activités théâtrales pour les atteindre sans qu'un produit soit au final présenté à un public extérieur.

Élargissant la notion de pédagogie du jeu dramatique, nous nous plaçons ici dans une pédagogie fondée sur le travail des professionnels du théâtre en amont des représentations voire pendant leur formation d'acteurs et entendons montrer que ces faire sociaux et professionnels peuvent donner lieu à des tâches complexes dans une approche interactionnelle de l'enseignement-apprentissage des langues. En cela, nous envisageons de dépasser l'usage qui est parfois fait de certains éléments issus du monde du théâtre et proposés, dans les manuels de langues ou des ouvrages consacrés au théâtre en classe de langues, dans le but d'apporter essentiellement une dimension poétique et/ou ludique au cours de langue. Plusieurs ouvrages présentent en effet des listes d'activités issues du monde du théâtre et utilisables dans le cadre de

5. Pour les questions et notamment les atouts liés au fait, pour les apprenants, de devenir acteurs, de se livrer à la mise en scène et de travailler parole et gestuelle, on se reportera à la thèse d'inspiration psycholinguistique de Pierra (1990) et à d'autres textes publiés par cette spécialiste du domaine et d'autres experts, notamment dans l'ouvrage collectif dirigé par Alix, Lagorgette et Rollinat-Levasseur (2013). 
l'enseignement-apprentissage (des langues) - c'est le cas par exemple de la première partie de la publication récente de Payet (2010); mais Schewe (1993, p. 34) ${ }^{6}$ note que, même dans ces ouvrages spécialisés ${ }^{7}$, les auteurs ne dépassent guère l'injection ponctuelle de telles activités sans tenter de «développer des unités didactiques dans lesquelles des formes d'activités dramatiques différentes se complètent pour faire sens ». Ainsi, la question du lien avec l'approche didactique générale se pose peu puisqu'on en reste au niveau de l'exercice.

Pourtant, de nombreuses activités complexes que réalisent les professionnels du théâtre au cours de leur formation ou dans le processus qui consiste à passer du texte écrit au texte représenté constituent des tâches potentiellement transposables dans le cadre du cours de langue. Reprendre ces tâches de la vie réelle (du théâtre) pour les intégrer dans un processus d'enseignement-apprentissage des langues permet d'associer didactique de la littérature, didactique du jeu dramatique et approche actionnelle voire interactionnelle, dans la mesure où les tâches comportent un enjeu social réel dépassant le seul apprentissage des langues. Cela permet en effet de proposer aux apprenants des «tâches "cibles", ou de "répétition" ou "proches de la vie réelle" (Conseil de l'Europe, 2001, p. 121), proches en cela de ce qui se fait dans le monde du théâtre.

\section{Approche interactionnelle au croisement de la théorie littéraire, de la didactique et de la pratique théâtrale}

Dans le domaine de la pratique théâtrale, nous nous intéresserons spécifiquement au travail des professionnels du théâtre qui fait que l'œuvre dramatique, écrite, limitée au système de signes que représente la parole et donc essentiellement conceptuelle, devient une œuvre scénique, sensible et sensorielle (Kowzan, 1969), exploitant plusieurs systèmes de signes. Nous nous situons ainsi au niveau du travail dramaturgique, autrement dit de l'activité d'interface entre le texte et le spectateur (Raoul-Davis, 1986; Dort, 1998; Danan, 2010). Ce travail visant le passage du texte à la représentation consiste en un «saut radical dans une dimension artistique différente» (Ryngaert, 2008, p. 22), en une

6. La pagination se réfère à la version électronique publiée en ligne dans les archives scientifiques ouvertes du University College Cork.

7. Il étudie trois ouvrages marquants du monde anglo- et germanophone : Dougill (1987), McRae (1985), Maley \& Duff (1981). 
«mutation de l'œuvre dramatique en œuvre scénique» (Dort, 1998). Il s'agit, pour reprendre les termes de Bernanoce (2013), de «l'art de penser et nourrir le passage à la mise en scène » (p. 32).

Concevant, comme Raoul-Davis ou encore Vitez (1991), que la dramaturgie constitue une activité qui n'est pas nécessairement dans les seules mains d'un «dramaturge», mais qui peut être partagée entre différents professionnels du théâtre, nous nous intéressons au travail de ceux-ci, qu'ils soient acteurs, metteurs en scène, décorateurs, costumiers, etc., ou, bien entendu, dramaturges. Ainsi, l'acteur est aussi «dramaturge à part entière quand il invente, avec l'assistance de tous les protagonistes de l'entreprise, le spectacle réel, le seul que les spectateurs verront jamais » (Raoul-Davis, 1986). Nous ne nous limitons pas au travail à la table, mais incluons le travail de plateau dans lequel nous voyons, à l'instar de Ryngaert (2008, p. 19), des «séances d'essai et de déchiffrement, de tâtonnements et d'expérimentations» permettant de «se livrer à divers modes d'essayage du texte en jeu».

$\mathrm{Si}$ le personnage est remis en cause par certains auteurs contemporains et si son étude reste complexe et les écueils nombreux (Ryngaert, 2008), le travail sur sa construction dans le domaine de la pratique théâtrale nous semble toutefois riche en tâches potentielles. Nous suivons Ryngaert (2008, p. 110) qui estime que le personnage reste une «nécessité irremplaçable et complexe de la fiction théâtrale». Tout comme le texte ne surgit qu'avec le travail du lecteur, «le personnage n'existe pas vraiment dans le texte, il ne se réalise que sur scène» (ibid., p. 110), fruit du travail de l'acteur voire du collectif de la troupe : «[1] es choix interviennent au moment du passage à la scène, quand une constellation d'acteurs se substitue à une constellation de fantômes» (ibid., p. 115). Reverdy (2014) pose à ce propos que «le lien "personnage-acteur", sans se poser comme définition [du théâtre], sert au moins de pivot pour ordonner l'ensemble des techniques qui font le théâtre» (p. 18).

S'il ne s'agit pas de tomber dans une approche purement psychologisante qui assimilerait le personnage à une personne, le travail de construction du personnage tel qu'il est pratiqué par de nombreux acteurs et a été insufflé par la pensée et la pratique de «théoriciens » tels que Stanislavski (1984 et 2001) et Strasberg (1969) présente un intérêt certain pour l'enseignement-apprentissage des langues, si, bien entendu, on l'applique à des pièces de théâtre qui s'y prêtent. Même si leurs orientations divergent en de nombreux points, Stanislavski et Strasberg conçoivent tous les deux le texte théâtral comme un texte incomplet que le travail et l'imagination de l'acteur (et du metteur en 
scène) - par extension tout travail dramaturgique - viennent enrichir pour accéder à une représentation. Rejoignant à sa manière les positionnements théoriques que nous évoquions plus haut, Stanislavski (1984) estime ainsi que «la pièce imprimée n'est pas une œuvre terminée tant qu'elle n'est pas jouée sur une scène par des acteurs et animée par des émotions humaines authentiques» (p. 139). En effet, l'auteur ne «fournit pas aux acteurs tout ce qu'ils doivent savoir de la pièce», il se montre même «souvent avare d'indications» sur les personnages, leurs sentiments, leurs pensées, leurs actes et «tout cela doit être rempli et approfondi par l'acteur» (Stanislavski, 2001, p. 77). La représentation surgit de la rencontre entre texte/auteur et acteurs :

Nous faisons vivre ce qui est caché derrière les mots, nous faisons passer nos propres pensées dans le langage de l'auteur, et nous établissons nos propres rapports avec les personnages de la pièce. Tous les matériaux que nous recevons de l'auteur et du metteur en scène se trouvent filtrés à travers notre personnalité, et complétés par notre imagination. (Ibid., p. 73)

Dans ce travail, l'imagination et donc la subjectivité sont sollicitées sans que le texte soit occulté. Ainsi, le travail artistique articule une «part de rêve» (Ryngaert, 2008, p. 120) à une lecture attentive du texte qui permet aux professionnels de faire des choix parmi les possibles. Dans un commentaire sur le travail de Strasberg, Hethmon ${ }^{8}$ (Strasberg, 1969) évoque précisément ce travail qui consiste à «faire fusionner la réalité du script et celle de l'acteur» (p. 276), autrement dit à faire se rapprocher ce que l'acteur développe dans son effort de vivre le personnage et la logique du texte de l'auteur.

Les acteurs évoquent souvent la nécessité de connaitre les personnages et de «s'impliquer passionnément dans le vie d'un autre», comme l'indique le titre d'une interview de Gert Voss dans le numéro 4 d'OutreScène (Benhamou, 2004a, p. 69). Ainsi, l'actrice Évelyne Didi, parlant de la relation aux personnages, précise :

Je peux m'identifier à eux, je peux les comprendre. [...] Les personnages sont tellement bien, tellement intéressants, même si leur vie est

8. L'ouvrage Le travail à l'Actors Studio publié sous le nom de Lee Strasberg a en fait été rédigé par R. Hethmon qui a recueilli des cours de Strasberg et alterne, dans l'ouvrage, des éléments attribués directement à Strasberg et des commentaires. 
nulle... [...] Si je fais du théâtre, c'est justement pour aller à la rencontre de ces gens-là. [...]

Il ne s'agit pas seulement de sentir les choses. Mais de creuser. D'apprendre à creuser. [...] On ne va pas se contenter de dire les choses justement, on va aller plus loin derrière, chercher comment elles sont arrivées là, pourquoi on dit ça. (Entretien dans Benhamou, 2004b, p. 23)

De cette nécessité de connaitre les personnages, de leur donner corps et vie découlent de nombreuses activités réalisées par les acteurs, le metteur en scène, mais aussi le costumier, le décorateur, etc. : il s'agit par exemple de donner une apparence physique au personnage, de construire sa vie intérieure, de l'imaginer dans différentes situations de la vie, de lui créer une histoire qui fait qu'il agit comme il le fait avec les autres personnages dans les situations présentées dans la pièce. Il s'agit également de créer les espaces dans lesquels il se trouve dans la pièce, de constituer sa garde-robe et de savoir ce qu'il portera dans les différentes scènes.

Ces activités du monde du théâtre représentent des tâches, au sens premier défini par Long (1985), des mille et une choses que l'on fait dans la vie de tous les jours, au travail, dans le jeu, etc. : " "Tasks" are the things people will tell you they do if you ask them and they are not applied linguists ${ }^{9}$.» (p. 89) Transposées dans le monde éducatif, elles correspondent aux tâches cibles (cf. supra) du fait qu'issues de la vie réelle, elles représentent un «plan de travail» guidé par une intention/un objectif, impliquent une priorité donnée au sens, des processus cognitifs et donnent lieu à un résultat communicatif clairement défini (voir notamment Ellis, 2003, p. 9 et suiv.; Guichon, 2006, p. 54; Ollivier \& Puren, 2011, p. 53).

\section{Mises en œuvre}

Nous revenons ici sur un exemple développé par nos soins dans le niveau 3 (B1) du manuel de FLE Version originale (Denyer, Ollivier \& Perrichon, 2011; désormais VO3), que nous enrichissons de quelques exemples complémentaires, afin de montrer concrètement comment une

9. «On entend par "tâche”, tout ce que les gens vous diront qu'ils font si vous leur demandez et s'ils ne sont pas des spécialistes de linguistique appliquée.» (Notre traduction) 
tâche issue du monde du théâtre peut être mise en œuvre dans un cours de langue et permettre d'allier, dans la perspective de réalisation de la tâche, des activités portant sur différentes activités langagières avec le travail des dimensions littéraire, linguistique et culturelle.

L'unité intitulée «La voix est le miroir de l'âme» vise un enregistrement de type radiophonique d'une pièce de théâtre d'Eugène Ionesco. La tâche présente ainsi un résultat concret : un enregistrement audio. L'intention est de partager avec les autres membres de la classe l'interprétation née du travail en petit groupe. L'ensemble va s'articuler autour de la tâche et des besoins langagiers, culturels, linguistiques, etc., qu'elle suscite.

Le travail en groupes permet de faire l'expérience du dialogisme et de la construction socio-interactionnelle de sens entre lecteurs dans la confrontation avec le texte de l'auteur. Les apprenants peuvent en effet prendre conscience, d'une part, des différentes façons de percevoir un texte ou des parties de texte et, d'autre part, du phénomène de «coopération interprétative» (cf. supra). Ils peuvent en outre se rendre compte que le texte est fondamentalement incomplet et requiert un travail de l'acteur pour l'interpréter.

Dans la construction d'une unité didactique centrée sur une tâche, il nous semble essentiel d'intégrer au maximum toutes les activités, de sorte que chacune contribue à la réalisation de la tâche. Dans le cadre d'une tâche issue du monde du théâtre, la possibilité s'offre aux concepteurs de proposer, en réception, des textes de spécialistes du théâtre évoquant le travail demandé aux acteurs dans le monde du théâtre et aux apprenants dans le cadre de la tâche. Il est également possible de proposer des textes informatifs sur l'auteur, l'époque où se déroule la pièce, les personnages (interview d'acteurs les ayant joués par exemple), etc. La réception de ces textes, constitutive du travail dramaturgique, doit alors être guidée par la perspective donnée par la tâche. Il s'agit d'en prendre connaissance pour en retirer des informations utiles pour réaliser ce qui est demandé. Dans le cas de l'unité 3 de $V O 3$, nous avons commencé par une activité de réception orale et écrite de textes de spécialistes du théâtre qui évoquent l'interprétation d'un texte : un texte de Stanislavski abordant la notion de «sous-texte», un autre sur ce qu'est ce sous-texte, un troisième sur la nécessité de bien connaitre les personnages avant de les jouer et finalement un extrait de Michail Chekhov lu pour la circonstance. Les apprenants sont alors invités à lire et écouter ces textes dans le but d'en retirer des conseils pour leur travail d'interprétation. 
Toujours dans cette même unité : une certaine connaissance de l'ancrage littéraire de Ionesco dans le théâtre de l'absurde permettant de mieux comprendre la scène proposée et donc d'en proposer plus aisément une interprétation qui soit issue de la rencontre entre l'auteur et ses lecteurs, des textes informatifs ont été proposés aux apprenants avec la consigne : «Qu'en retenez-vous pour la lecture de la courte pièce d'Eugène Ionesco dans la tâche?» (Ibid., p. 44) La dimension culturelle s'intègre ainsi au sein même de la tâche.

À noter que la dimension interculturelle pourra également être fortement présente dans la réalisation de tâches issues du monde du théâtre. Ce sera par exemple le cas pour une tâche demandant de décrire la façon dont on voit un personnage lorsqu'elle sera réalisée dans un groupe multiculturel : en fonction des cultures en présence, on pourra avoir des perceptions différentes d'un même personnage ou avoir une image partagée de son caractère, mais transcrire celle-ci en l'habillant de manières différentes. Il peut alors s'avérer intéressant de recueillir et discuter les façons dont sont perçus culturellement les différents «costumes».

Nous abordons maintenant la dimension linguistique de l'apprentissage en lien avec la tâche. La tâche présentée ci-dessus demandant une interprétation orale d'un texte théâtral, nous avons proposé aux apprenants des activités que font les acteurs : à savoir l'ajout d'indications de jeu sur le texte en lien avec la notion de sous-texte et un travail sur la façon dont on s'imagine les personnages et tout particulièrement les regrets et les espoirs du personnage principal afin de mieux en appréhender les motivations. Cela a permis d'introduire un travail sur les adjectifs et adverbes exprimant des sentiments, sur l'expression du regret et de l'espoir et de réactiver des connaissances sur les vêtements. En outre, un travail d'entrainement à l'expression de sentiments a été proposé, issu lui aussi du monde du théâtre : identifier et exprimer plusieurs sentiments à l'aide d'un même énoncé.

Si l'on reprend les propos de Stanislavski sur la construction du personnage, on se rend compte du nombre important de tâches possibles :

Vous devez l'étudier du point de vue de son époque, des circonstances, de son pays, de ses conditions de vie, de son entourage, de la littérature, de la psychologie, de son esprit, de sa façon de vivre, de sa position sociale, de son aspect extérieur; également de ce qui le caractérise, sa personnalité propre, c'est-à-dire ses habitudes, ses manières, ses attitudes, sa voix, sa façon de s'exprimer, ses intonations... C'est en travaillant ainsi que vous parviendrez à l'animer de vos propres sentiments. Sans tout cela, il n'y a pas d'art. (Stanislavski, 2001, p. 37) 
Ces propos de Stanislavski invitent, par exemple, à travailler, à partir de pièces ou d'extraits pertinemment choisis pour susciter l'imagination, la description physique et morale, le récit de vie, la présentation d'une journée-type, l'évocation de l'éducation, des peurs et espoirs... de différents personnages. Et pour prendre un autre exemple plus lié au travail du metteur en scène et du décorateur, imaginer la scène où se déroule l'action de la pièce ou l'environnement dans lequel habite un personnage permet de travailler la description de lieux. On retrouve là de nombreux éléments fréquemment présents dans les manuels de langue.

Tout cela demande, à l'acteur et à l'apprenant devant réaliser une tâche issue du monde du théâtre, un travail intérieur suivi d'une «manifestation extérieure» (ibid., p. 31). Au théâtre, selon les approches et les personnes, il pourra s'agir d'activités concrètes non langagières et/ou de manifestations langagières. Dans La construction du personnage, Tortsov (qui représente Stanislavski dans cet écrit théorique dans lequel l'auteur fait agir des élèves-acteurs et leur professeur, Torstov) convie ses étudiants-acteurs à «préparer l'aspect extérieur d'un personnage, s'en revêtir, et [...] le présenter» (Stanislavski, 1984, p. 26) au reste du groupe. Cela donne lieu à des activités différentes selon les élèves, quelques-unes ayant une dimension langagière, d'autres (les plus nombreuses) non : «Chacun de nous commença à penser à quelque chose, à imaginer quelque chose, à prendre des notes, à tracer des dessins jalousement cachés, en fonction d'un portrait, d'un costume, d'un maquillage.» (Ibid.) Dans le cadre de la classe de langue, où il s'agit bien évidemment de développer, entre autres, la dimension langagière, on demandera essentiellement aux apprenants des activités orales ou écrites permettant de partager et comparer leurs différentes représentations intérieures sans empêcher ceux qui le souhaiteront de passer par des activités non langagières (dessins par exemple si on reprend l'exemple que nous venons de citer). Dans le cas de l'unité 3 de VO3, la tâche faisant en soi appel à une dimension langagière, nous avons pu la proposer telle que dans le monde du théâtre.

\section{Retour sur approche et perspectives}

Nous nous sommes attaché à montrer qu'il est possible, dans une optique de croisement des mondes, de construire des unités didactiques cohérentes autour de tâches théâtrales articulant des activités issues du travail de l'acteur. Il est possible, en ayant recours à des tâches issues 
du monde du théâtre, de viser de façon intégrée des objectifs de lecture littéraire comprise comme co-construction de sens entre auteur et lecteur, mais aussi entre lecteurs, des objectifs langagiers et linguistiques précis, des objectifs culturels voire interculturels et une découverte du monde du théâtre.

Ces tâches s'inscrivent pleinement dans une perspective actionnelle, voire dans une approche interactionnelle de l'enseignement-apprentissage des langues qui prend pleinement en compte les dynamiques sociales en présence. Elles s'effectuent en effet au sein d'interactions sociales et demandent une implication du sujet, chaque apprenant étant invité à partager avec ses pairs et l'enseignant sa vision personnelle du personnage, de la scène, etc. L'apprenant est acteur de la co-construction de sens et fait l'expérience du dialogisme de celle-ci en ayant accès aux lectures de ses pairs qui lui permettent de prendre conscience que toutes les lectures ne sont pas semblables car issues de co-énonciateurs différents.

Dans toutes ces situations, «les apprenants ne sont pas amenés à adopter un statut social imaginaire ni à se considérer dans une configuration spatio-temporelle différente de celle dans laquelle ils se trouvent réellement» (Pakdel, 2011, p. 125), ils parlent en leur nom au sein des interactions sociales en présence, ils utilisent le langage pour partager leurs représentations personnelles intérieures. Ils agissent hic et nunc en qualité de sujets engagés dans des activités issues du monde hors institution d'apprentissage, dans notre cas, celui des professionnels du théâtre.

\section{Remerciements}

L'auteur de ce texte tient à remercier le relecteur ou la relectrice qui a formulé, dans ses retours, des pistes constructives qui ont permis d'enrichir cette contribution.

\section{RÉFÉRENCES BIBLIOGRAPHIQUES}

Alix, Christophe, Lagorgette, Dominique \& Rollinat-Levasseur, ÈveMarie (dir.). (2013). Didactique du français langue étrangère par la pratique théâtrale. Chambéry : Université de Savoie, Laboratoire LLS.

BaKhtine, Mikhaïl M. (1978). Esthétique et théorie du roman (D. Olivier, trad.). Paris : Gallimard. (Ouvrage original publié en 1975 sous le titre 
Voprosy literatury i estetiki. Moscou, Russie : Khudozhestvennaia literatura).

BAKhtine, Mikhaïl M. (1984). Esthétique de la création verbale (A. Aucouturier, trad.). Paris : Gallimard. (Ouvrage original publié en 1979 sous le titre Estetika slovesnogo tvorchestva. Moscou, Russie : Iskusstvo).

B ARThes, Roland. (1973). Le plaisir du texte. Paris : Seuil.

Barthes, Roland. (1994). Euvres complètes (tome 2). Paris : Seuil.

BAYARD Pierre (1998). Qui a tué Roger Ackroyd? Paris : Minuit.

Bemporad, Chiara (2010). Le Cadre et la littérature. Proposition d'une articulation possible. Dans $11^{\text {es }}$ rencontres des chercheurs en didactique des littératures (p. 19-24), Genève. Disponible en ligne sur <www.unige. ch/litteratures2010/contributions_files/Bemporad 2010.pdf $>$ (consulté le 21 avril 2015).

Benhamou, Anne-Françoise. (2004a). L'acteur et son rôle, OutreScène, 4. Strasbourg : Théâtre national de Strasbourg.

Benhamou, Anne-Françoise (2004b). Le rôle de l'acteur, OutreScène, 3. Strasbourg : Théâtre national de Strasbourg.

Benveniste, Émile. (1974). Problèmes de linguistique générale. Paris : Gallimard.

Bernanoce, Marie. (2013). Le répertoire théâtral dans son contexte scolaire, à l'épreuve des genres et des esthétiques. Le français aujourd'hui, 180, 27-38.

Bolton, Gavin M. (1979). Towards a Theory of Drama in Education. Londres, Grande-Bretagne : Longman.

Bolton, Gavin M. (1984). Drama as Education: An Argument for Placing Drama at the Centre of the Curriculum. Harlow, Essex, GrandeBretagne : Longman.

Bredella, Lothar. (1980). Das Verstehen literarischer Texte. Stuttgart, Berlin, Cologne, Mayence, Allemagne : Kohlhammer.

Chrifi Alaoui, Dalle. (2007). Apports humanistes et linguistiques des pratiques artistiques dans l'apprentissage et l'enseignement de la langue dans une classe d'accueil. Études de linguistique appliquée, 147(3), 349-364. Disponible en ligne sur <http://www.cairn.info/article.php? ID_ARTICLE=ELA_147_0349> (consulté le 21 avril 2015).

Conseil de L'Europe. (2001). Cadre européen commun de référence pour les langues : apprendre, enseigner, évaluer. Paris : Didier; Strasbourg : Conseil de l'Europe. Disponible en ligne sur <http://www.coe.int/t/dg4/ linguistic/Source/Framework_fr.pdf> (consulté le 21 avril 2015).

Cornaz, Sandra \& Fonio, Filippo. (2014). Présentation et premiers résultats de la conception d'un référentiel de compétences en pratiques artistiques et apprentissage des langues. Dans J. Aden \& A. Arleo (dir.), Actes de colloque : "Langues en mouvement : didactique des langues 
et pratiques artistiques» (E-Crini, $\left.\mathrm{n}^{\circ} 6\right)$. Nantes : Éditions du CRINI. Disponible en ligne sur <www.crini.univ-nantes.fr/1403000125802/0/ fiche_pagelibre/\&RH=1402999468883> (consulté le 21 avril 2015). Culioli, Antoine. (1999). Pour une linguistique de l'énonciation. Tome 2 : Formalisation et opérations de repérage. Gap, Paris : Ophrys.

Danan, Joseph. (2010). Qu'est-ce que la dramaturgie? Arles : Actes Sud. Denyer, Monique, Ollivier, Christian \& Perrichon, Émilie. (2011). Version originale 3. Méthode de français. Livre de l'élève. Paris : Éditions Maison des langues.

Derrida, Jacques. (1967). L'écriture et la différence. Paris : Seuil.

Dort, Bernard. (1998). Dramaturgie. Dans Dictionnaire encyclopédique du théâtre (vol. 1, p. 520-522). Paris : Larousse.

DougiLl, John. (1987). Drama Activities for Language Learning. Londres, Grande-Bretagne : Macmillan.

Eco, Umberto. (1983). Postille a «Il nome della rosa». Alfabeta, 49. Disponible en ligne sur <www.epubbud.com/read.php?g=QZSX5QYA\&p $=66>$ (consulté le 21 avril 2015).

Eco, Umberto. (2013). Confessions d'un jeune romancier (F. Rosso, trad.). Paris : Grasset. (Ouvrage original publié en 2011 sous le titre Confessions of a Young Novelist. Cambridge, États-Unis : Harvard University Press).

Eichmann, Walter. (1990). Produktions- und handlungsorientierter Literaturunterricht. Bad Kreuznach, Allemagne : PZ.

ELlis, Rod. (2003). Task-Based Language Learning and Teaching. Oxford, Grande-Bretagne; New York, États-Unis : Oxford University Press.

Fonio, Filippo \& Genicot, Geneviève. (2011). The Compatibility of Drama Language Teaching and CEFR Objectives - Observations on a Rationale for an Artistic Approach to Foreign Language Teaching at an Academic Level. Scenario. Journal for Drama and Theatre in Foreign and Second Language Education, 5(2). Disponible en ligne sur <http://research.ucc. ie/scenario/2011/02/FonioGenicot/06/en> (consulté le 21 avril 2015).

Foucault, Michel. (1969). L'archéologie du savoir. Paris : Gallimard.

GADAMER, Hans-Georg. (1975). Wahrheit und Methode : Grundzüge einer philosophischen Hermeneutik. Tübingen, Allemagne : Mohr.

GrILlo, Éric. (1997). La philosophie du langage. Paris : Seuil.

GRILlo, Éric. (2000). Intentionnalité et signifiance : une approche dialogique. Bern, Suisse; Berlin, Allemagne; New York, États-Unis : Peter Lang.

Gudjons, Herbert. (1986). Handlungsorientiert lehren und lernen: Projektunterricht und Schüleraktivität. Bad Heilbrunn, Allemagne : Klinkhardt.

Guichon, Nicolas. (2006). Langues et TICE : méthodologie de conception multimédia. Paris : Ophrys. 
HAAs, Gerhard. (2005). Handlungs- und produktionsorientierter Literaturunterricht: Theorie und Praxis eines ,anderen“ Literaturunterrichts für die Primar- und Sekundarstufe. Seelze-Velber, Allemagne : Kallmeyer.

IsER, Wolfgang. (1976). Der Akt des Lesens: Theorie ästhetischer Wirkung. Munich, Allemagne : W. Fink.

JACQUES, Francis. (1979). Dialogiques : recherches logiques sur le dialogue. Paris : Presses universitaires de France.

JACQUES, Francis. (1982). Le schéma jakobsonien de la communication est-il devenu un obstacle épistémologique? Dans N. Mouloud \& P. Vienne (dir.), Langages, connaissance et pratique (p. 157-184). Lille : Presses universitaires du Septentrion.

JACQues, Francis. (1985). Dialogiques. Tome 2 : L'espace logique de l'interlocution. Paris : Presses universitaires de France.

JACQUES, Francis. (2000). Écrits anthropologiques. Philosophie de l'esprit et cognition. Paris : L'Harmattan.

Jouve, Vincent. (2004). La lecture comme retour sur soi : de l'intérêt pédagogique des lectures subjectives. Dans A. Rouxel et G. Langlade (dir.), Le sujet lecteur. Lecture subjective et enseignement de la littérature (p. 105-114). Rennes : Presses universitaires de Rennes.

Kowzan, Tadeusz. (1969). Le texte et le spectacle. Rapports entre la mise en scène et la parole. Cahiers de l'Association internationale des études françaises, 21, 63-72.

LANGlade, Gérard. (2008). Activité fictionnalisante du lecteur et dispositif de l'imaginaire. Figura, 20, 46-65.

LANGlade, Gérard. (2004). Sortir du formalisme, accueillir les lecteurs réels. Le français aujourd'hui, 145(2), 85-96.

Long, Michael H. (1985). A Role for Instruction. Dans K. Hyltenstam \& M. Pienemann (dir.), Modelling and Assessing Second Language Acquisition (p. 77-99). San Diego, États-Unis : Multilingual Matters.

Luscher, Jean-Marc. (2009). L'enseignement de la littérature selon la perspective actionnelle. Que pourrait être une «tâche littéraire»? Le français à l'université, 14(2). Disponible en ligne sur <http://eprints.aidenlignefrancais-universite.auf.org/137/> (consulté le 21 avril 2015).

Maley, Alan, Duff, Alan \& Freudenstein, Reinhold. (1981). Szenisches Spiel und freies Sprechen im Fremdsprachenunterricht : Grundlagen und Modelle für die Unterrichtspraxis. Munich, Allemagne : Hueber.

Mallarmé, Stéphane. (1897). Divagations. Crise de vers. Paris : Gallimard. McRAe, John. (1985). Using Drama in the Classroom. Oxford, GrandeBretagne; New York, États-Unis : Pergamon Institute of English.

Menzel, Wolfgang. (2000). Handlungsorientierter Literaturunterricht. Seelze, Allemagne : Friedrich. 
Morel, Sandra. (2014). Jeux de mots, jeux de gestes. Pour une «mimodynamique fictionnaire». Dans J. Aden \& A. Arleo (dir.), Actes de colloque : "Langues en mouvement : didactique des langues et pratiques artistiques $\gg\left(E-C r i n i, \mathrm{n}^{\circ} 6\right)$. Nantes : Éditions du CRINI. Disponible en ligne sur <www.crini.univ-nantes.fr/ $1403000125802 / 0 /$ fiche__page libre/\&RH=1402999468883> (consulté le 21 avril 2015).

Ollivier, Christian. (2009). La vie après la vie : approche interactionnelle et co-construction de sens sur un blog littéraire. Dans C. Develotte, F. Mangenot \& E. Nissen (dir.), Actes du colloque «Échanger pour apprendre en ligne» $(E P A L)$. Disponible en ligne sur $<\mathrm{http}: / / \mathrm{w} 3 . \mathrm{u}-$ grenoble3.fr/epal/actes.html> (consulté le 21 avril 2015).

OlLIVIER, Christian. (2010). Interactions et collaboration interprétative sur le web 2.0. Littérature et plaisir de lire, Les Langues Modernes, 3. Disponible en ligne sur <www.aplv-languesmodernes.org/spip.php? article3353> (consulté le 21 avril 2015).

Ollivier, Christian \& Puren, Laurent. (2011). Le web 2.0 en classe de langue. Paris : Éditions Maison des langues.

PaKdel, Ali. (2011). De l'activité communicative à l'activité sociale d'apprentissage des langues en ligne : analyse de la dynamique sociale en contexte institutionnel (Thèse de doctorat, Université d'Aix-Marseille, Aix-en-Provence/Marseille). Disponible en ligne sur $<$ http://tel.archivesouvertes.fr/tel-00637137/> (consulté le 21 avril 2015).

PAYET, Adrien. (2010). Activités théâtrales en classe de langue. Paris : CLE International.

Pennac, Daniel. (1992). Comme un roman. Paris : Gallimard.

PIERRA, Gisèle (1990). Le théâtre dans l'enseignement du français langue étrangère. L'anti-méthode. Montpellier : Université Paul-Valéry Montpellier 3 .

PUREN, Christian. (2006). Explication de textes et perspective actionnelle: la littérature entre le dire scolaire et le faire social. Site de l'APLV. Disponible en ligne sur <www.aplv-languesmodernes.org//spip.php? article389> (consulté le 21 avril 2015).

Puren, Christian. (2012). Perspectives actionnelles sur la littérature dans l'enseignement scolaire et universitaire des langues-cultures : des tâches scolaires sur les textes aux actions sociales par les textes. Disponible en ligne sur $<$ www.christianpuren.com/mes-travaux/2012d/> (consulté le 21 avril 2015).

Raoul-Davis, Michèle. (1986). Profession «dramaturge». Théâtre/Public, 67, 4-6.

REvERDY, Marie. (2014). La dramatique vie de marie r. Offshore. Art contemporain, $34,18$. 
RouXEL, Annie. (2010). L'appropriation singulière de la culture littéraire comme fondement d'une identité francophone vivante. Dans J.-P. Cuq et P. Chardenet (dir.), Faire vivre les identités : un parcours en francophonie (p. 51-60). Paris : Archives contemporaines.

Rouxel, Annie. (2007). Pratiques de lecture : quelles voies pour favoriser l'expression du sujet lecteur? Le français aujourd'hui, 157(2), 65-73. Disponible en ligne sur <www.cairn.info/revue-le-francais-aujourd-hui2007-2-page-65.htm> (consulté le 21 avril 2015).

RYNGAERT, Jean-Pierre. (2008). Introduction à l'analyse du théâtre. Paris : A. Colin.

SARTre, Jean-Paul. (1948). Qu'est-ce que la littérature? Paris : Gallimard. Schewe, Manfred. (1993). Fremdsprache inszenieren: zur Fundierung einer dramapädagogischen Lehr- und Lernpraxis. Oldenburg, Allemagne : Zentrum für pädagogische Berufspraxis, Universität Oldenburg. Disponible en ligne sur <https://cora.ucc.ie/handle/10468/561> (consulté le 21 avril 2015).

SchleIERMACHER, Friedrich. (1843). Die christliche Sitte. Berlin, Allemagne : Jonas.

Spanghero-Gaillard, Nathalie \& Garnier, Emmanuelle (dir.). (2014). Pratiques théâtrales en classe de langue, Les Langues Modernes, 4. Paris : APLV.

Stanislavski, Constantin. (1984). La construction du personnage (C. Antonetti, trad.). Paris : Pygmalion / Gérard Watelet. (Ouvrage original publié en 1949 sous le titre Building a Character. New York, États-Unis : Theatre Arts).

Stanislavski, Constantin. (2001). La Formation de l'acteur (É. Janvier, trad.). Paris : Payot \& Rivages. (Ouvrage original publié en 1936 sous le titre An Actor Prepares. New York, États-Unis : Theatre Arts).

Strasberg, Lee. (1969). Le travail à l'Actors studio (D. Minot, trad.). Paris : Gallimard. (Ouvrage original publié en 1965 sous le titre Strasberg at the Actors Studio. New York, États-Unis : Viking Press).

TAuveron, Catherine. (2004). La lecture comme jeu, à l'école aussi. Dans C. Tauveron (dir.), La lecture et la culture littéraires au cycle des approfondissements : actes de l'université d'automne (p. 23-40). CRDP de Versailles. Disponible en ligne sur <http://media.eduscol.education.fr/ file/Formation_continue_enseignants/14/7/lecture_culture_litteraires_ 111147.pdf> (consulté le 21 avril 2015).

VAléry, Paul. (1960). Euvres (tome 2). Paris : Gallimard.

VAlÉRY, Paul. (1933). Avant-propos. Dans G. Cohen, Essai d'explication du Cimetière marin (p. 7-36). Dijon : Darantière. 
VITEZ, Antoine. (1991). Sur le dramaturge, réponse à un questionnaire de l'ATAC (Association technique de l'action culturelle). Dans Le Théâtre des Idées, anthologie proposée par Danielle Sallenave et Georges Banu. Paris : Gallimard. 